\title{
Image Processing based Multilingual Translator for Travellers using Raspberry pi
}

\author{
S. Rishi Kumar ${ }^{1}$, G.Madhavan ${ }^{1}$, M. Naveen ${ }^{1}$, S.Subash ${ }^{1}$, U. Selvamalar Beulah Ponrani ${ }^{2}$ \\ Final Year Students, Dept of Electrical and Electronics Engineering, Velammal Engineering College, Chennai, India ${ }^{1}$ \\ Faculty Advisor, Dept of Electrical and Electronics Engineering, Velammal Engineering College, Chennai, India ${ }^{2}$
}

\begin{abstract}
The present paper is about an innovative, efficient and real-time cost beneficial method that enables international travelers to hear the text images of sign boards, routes in their own languages. It combines the concept of Optical Character Recognition (OCR), text to Speech Synthesizer (TTS) and translator in Raspberry pi. Text Extraction from color images is a challenging task in computer vision. Text-to-Speech conversion is a method that scans and reads any language letters and numbers that are in the image using OCR technique and then translates it into any desired language and at last it gives audio output of the translated text. The translate shell which is used to translate the text to is available in many languages. The translate shellis utilized to provide audio output too. This paper describes the design, implementation and experimental results of the device. This device consists of two modules, image processing module and voice output module. The device was developed based on Raspberry Pi b+ with $900 \mathrm{MHz}$ processor speed.
\end{abstract}

Keywords: Translator, OCR, Rassberry pi, Tesseract , image magic, python

\section{INTRODUCTION}

Optical character Recognition (OCR) is a process that Then, it takes a picture; a delay of around 7 seconds is converts scanned or printed text images, handwritten text provided, which helps to focus the webcam, if it is into editable text for further processing[1]. This paper accidently defocused. After delay, picture is taken and presents a robust approach for text extraction and processed by Raspy to hear the translated words of the text converting it to speech. Testing of device was done on through the earphone or speaker plugged into Raspy raspberry pi platform. The Raspberry $\mathrm{Pi}$ is a basic through its $3.5 \mathrm{~mm}$ audio jack. embedded system and being a low cost a singleboard computer used to reduce the complexity of systems in real time applications. This platform is mainly based on python. Raspberry pi consist of Camera slot Interface (CSI) to interface the raspberry pi camera. Here, the Dark and Low contrast images captured by using the Raspberry Pi camera module are enhanced in order to identify the particular region of image. The Raspy is initially connected to the internet through wireless dongle. The software is installed using command lines. Following steps are to be followed:

1. The first setup is to download the installation script.

2. Second step is to convert it to executable form.

3. The last step starts the script which does the rest of the installation work.

Device set up is done as shown in Figure. 1a. The webcam is manually focused towards the text.

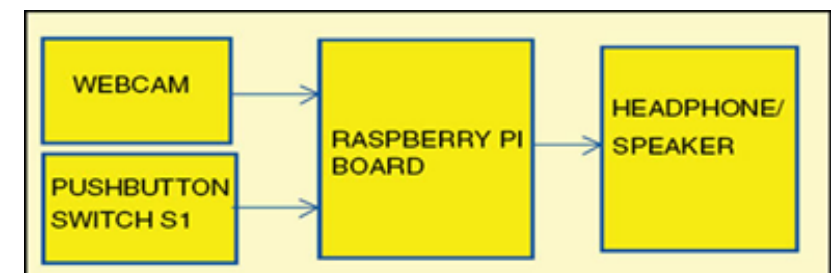

Fig. 1aBlock diagram of Image processing based multilingual translator.

\section{BASIC CONCEPT OF IMAGE PROCESSING BASED TRANSLATION}

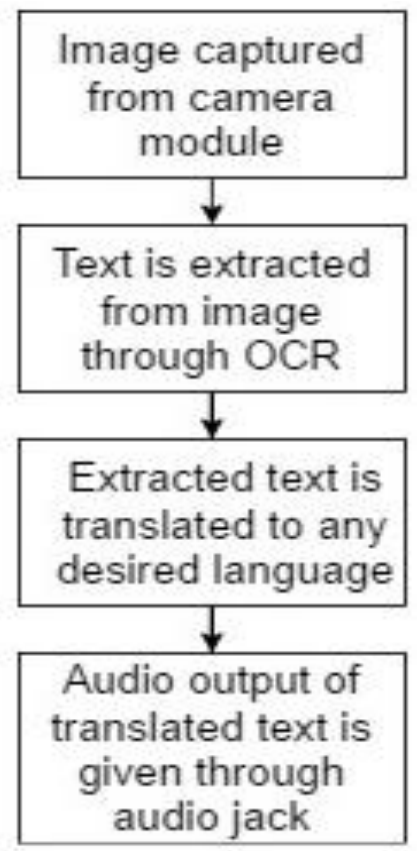

Fig. 1b.Procedural flow of image processing based translation 
In general, any digital image processing algorithm consists is based on the Broadcom BCM2835 system on a chip of three stages: input, processor and output. In the input (SOC) which includes an ARM1176JZF-S Core (ARM stage image is captured by a camera. It sent to a particular V6K)700 MHz CPU processor, Broadcom Video Core IV system to focus on a pixel of image that's gives, its output GPU having 17 pins, 3.5W of power, and $512 \mathrm{MB}$ of as a processed image. This processed image is then used to RAM memory. This main processing chip connects a translate after extracting the text from the image through camera and display. The Raspberry Pi design does not OCR.

\section{SYSTEM HARDWARE DESIGN}

The Raspberry Pi board is the central module of the whole embedded image capturing and processing system as given in figure. 2. Its main parts include: main processing chip unit, memory, power supply HDMI Out i.e. VGA display, Ethernet port, and USB ports. The webcam is connected to Raspberry pi through one of its USB ports and pushbutton switch S1 to its GPIO pin 16 (or GPIO23) through resistor R2 (1-kilo-ohm)

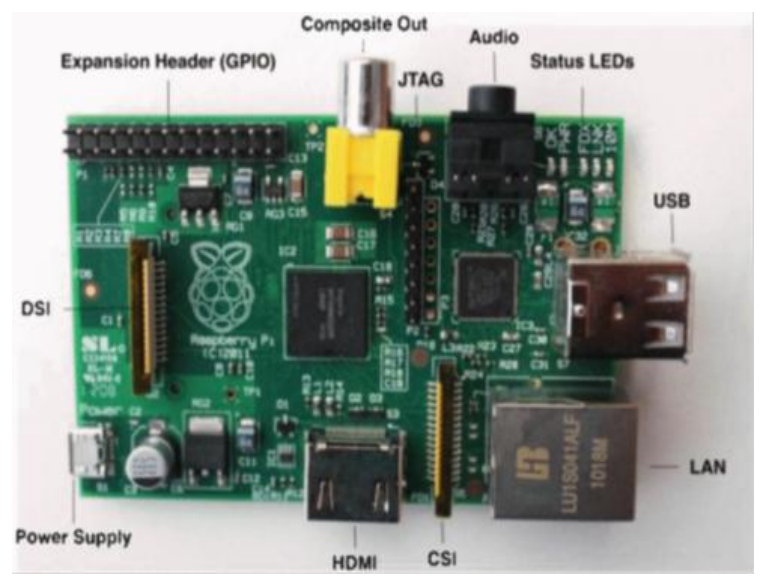

Fig. 2: Raspberry Pi board (Model B+).

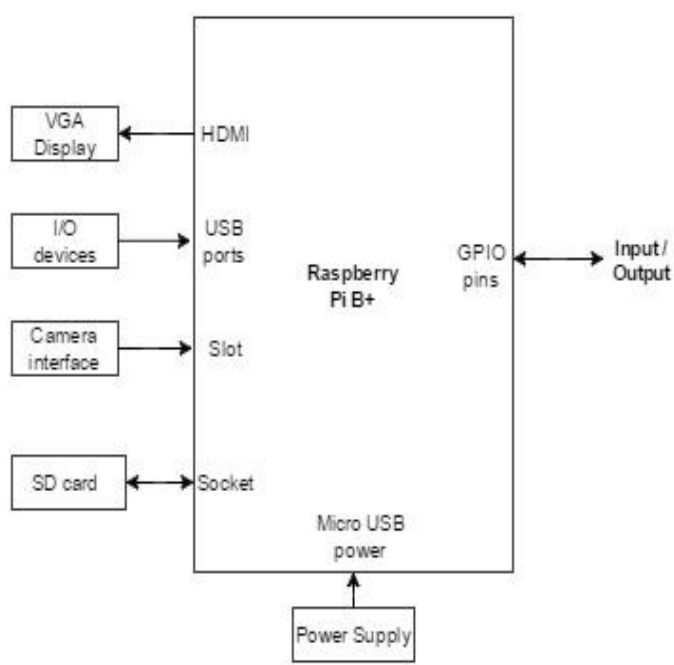

Fig. 3: System Block Diagram

A.Raspberry pi board

The Raspberry $\mathrm{Pi}$ is a basic embedded system having a credit card-sized single board computers developed in the UK by the Raspberry Pi Foundation[2]. The Raspberry Pi include a built in hard disk or solid state drive, instead used an SD card for booting and long term storage. This board is intended to run Linux Debian based operating systems. Python is used as main programming language for raspberry pi .This Raspberry Pi module has a Samsung class 4 micro SD card preloaded with the Raspberry Pi NOOBS (New out of Box Software) package, and a printed Micro SD card adaptor.

\section{B. Camera interface}

The camera module used in this paper is raspberry picamera module as shown in the Figure. 3. The camera module plugs to the CSI connector on the Raspberry Pi. It's able to deliver clear 5MP resolution image, or $1080 \mathrm{p}$ HD video recording at $30 \mathrm{fps}$. The camera module attaches to Raspberry Pi by a 15 pin Ribbon Cable, to the dedicated 15 pin MIPI Camera Serial Interface (CSI), which was designed especially for interfacing to cameras. The CSI bus is capable of extremely high data rates, and it exclusively carries pixel data to the BCM2835 processor.

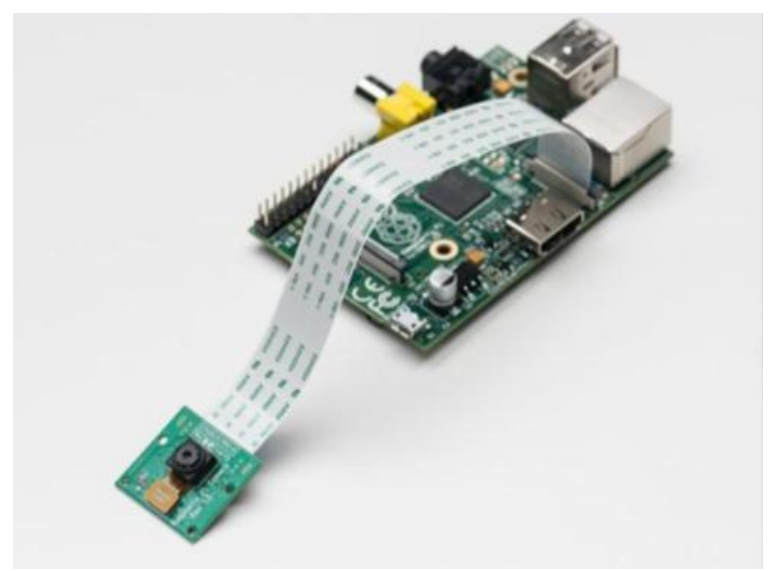

Fig. 4: Raspberry Pi camera board

C. Pushbutton switch set up

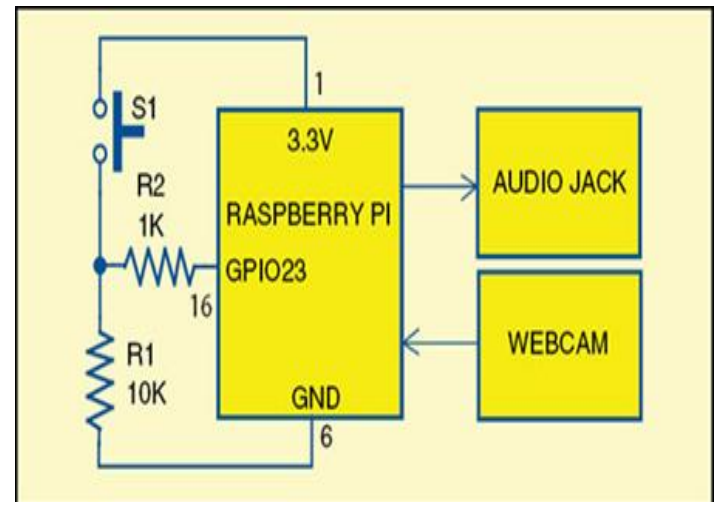

Fig.5 Circuit connection to Raspberry pi board. 
In Figure.5, When the GPIO pin is set as input, it is floating and has no defined voltage level, to detect whether the input is high or low, simple resistive circuit is needed so that it is always connected and reads either high or low voltage. One of the terminals of switch $\mathrm{S} 1$ is connected to ground (GPIO pin 6) through pull-down resistor R1 of 10 kilo ohm. The other terminal is connected to $3.3 \mathrm{~V}$ of GPIO pin 1 . When $\mathrm{S} 1$ is pressed, a high voltage is read on GPIO pin 16 . When $\mathrm{S} 1$ is released, GPIO pin 16 is connected to ground through $\mathrm{R} 1$, hence a low voltage is read by GPIO pin 16 . When pushbutton S1 is pressed, the webcam takes a picture of the text (after a delay of 10 seconds).

\section{METHODOLOGY}

The device consists of two main modules, the image processing module and translator module (Figure.6). Image processing module captures image using camera, converting the image into text. Translate module translates the text into any desired language and processes it with specific physical characteristics so that the sound can be understood. Figure 6 shows the block diagram of the device, 1st block is image processing module, where OCR converts .jpg to .txt form. 2nd is voice processing module which translates and gives .txt to speech output.

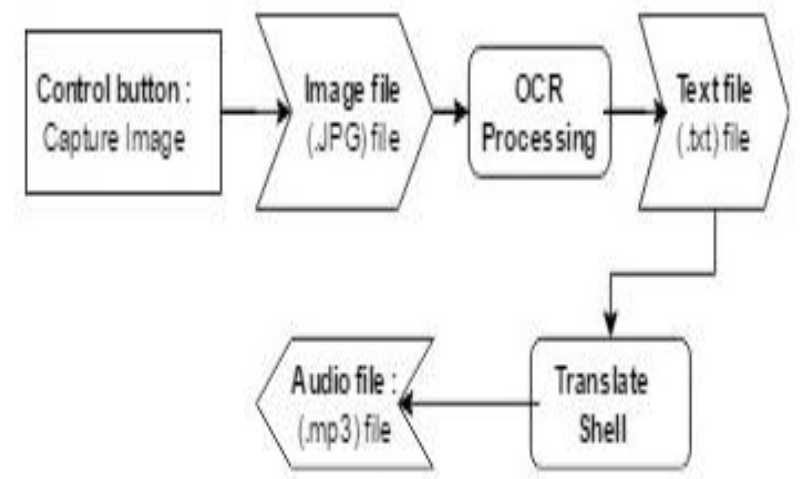

Fig.6 Image processing and translator modules

Figure 6 shows the block diagram of the device, 1st block is image processing module, where OCR converts .jpg to .txt form. 2nd is translate module which translates the text and gives audio output.

\section{IV.(i) IMAGE PROCESSING MODULE USING OPTICAL CHARACTER RECOGNITION}

OCR is important element in this module. OCR or Optical Character Recognition is a technology that automatically recognize the character through the optical mechanism, this technology imitate the ability of the human senses of sight, where the camera becomes a replacement for eye and image processing is done in the computer engine as a substitute for the human brain [3]. Tesseract OCR is a type of OCR engine with matrix matching [4]. The selection of Tesseract engine is because of its flexibility and extensibility of machines and the fact that many communities are active researchers to develop this OCR engine and also because Tesseract OCR can support 149 languages. In this project weare identifying English alphabets. Before feeding the image to the OCR, it isconverted to a binary image to increase the recognition accuracy. Image binary conversion is done by using Imagemagick software, which is another open source tool for image manipulation. The output of OCR is the text, which is stored in a file (speech.txt) .Machines still have defects such as distortion at the edges and dim light effect, so it is still difficult for most OCR engines to get high accuracy text [5]. It needs some supporting and condition in order to get the minimal defect.

a.Tesseract OCR Implementation

The input image is captured by the 5 megapixel fixed focus raspberry pi camera which supports 720p60, 1080 p30 video modes. Based on the specifications of the Tesseract OCR engine, the minimum character size that can be read is 20 pixels uppercase letters. Tesseract OCR accuracy will decrease with the font size less than $14 \mathrm{pt}$.

\section{b.Software Design}

Software processes the input image and convert it into text format. Then the text is translated into any desired language. The software implementation is showed in Figure. 7.The image is taken by the user via GPIO pin (23) that is connected to the button, using interrupt function. Furthermore, the picture cantaken by using raspistillprogram with sharpness mode to sharpen the image. The resulting image has a .jpg format with a resolution of $720 \times 340$ pixels.

\section{c. The Translate Module}

In this module text is converted to text of desired language. The output of OCR is the text, which is stored in a file (speech.txt). Here, Translate Shell software is used to convert the text to translated speech output. Translate Shell is an open source translate (TTS) system, which is available in many languages. In this project, English TTS system is used for reading the text.

\section{DESIGN IMPLEMENTATION}

The testing was done using Raspberry Pi platform with the following specifications:

- SBU Raspberry Pi 2900 MHz Quad Code ARM CortexA7

- 5mp Raspberry pi b+ camera module

- Bootable SanDisk Ultra 8GB micro SD Card

\section{Steps Followed:}

1. Import and Initialization:

Import subprocess, time and RPi.GPIO and initialize GPIO pin 23 as input.

\section{Main Program:}

The main program [6]provides functions to retrieve and process the input image, translate it and convert it into an 
audio signal. Picture will be taken as soon as push button switch is pressed then this Captured image is threshold before feeding it to OCR to increase the accuracy. The text from the captured image is extracted. The extracted text is translated to desired language and given out as audio signal. Overall flow of program is done as in flowchart fig 7.

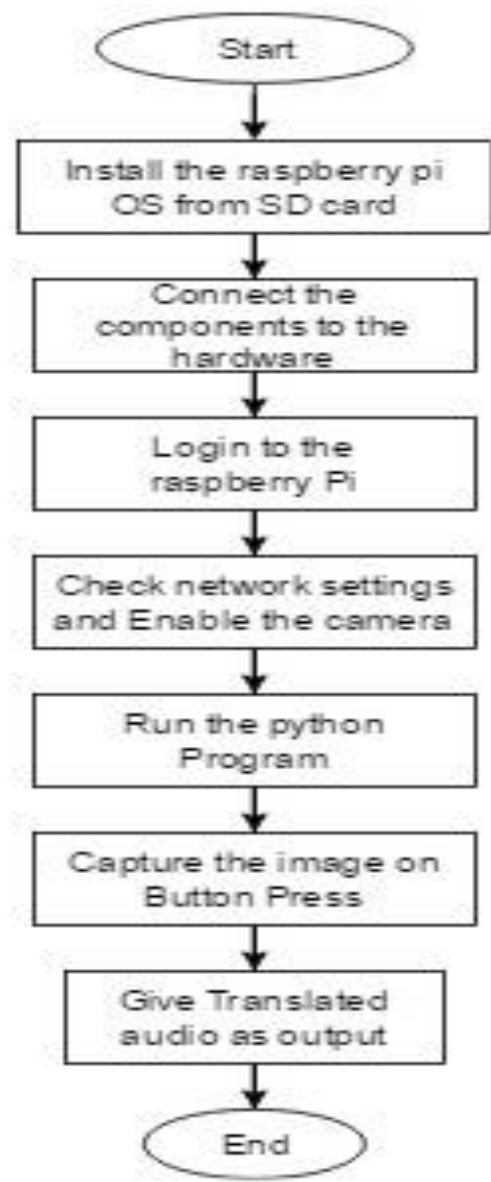

Fig.7 Flow chart of Image processing based translator device program

Screenshot Program execution for english to spanish translation is shown in figure 8

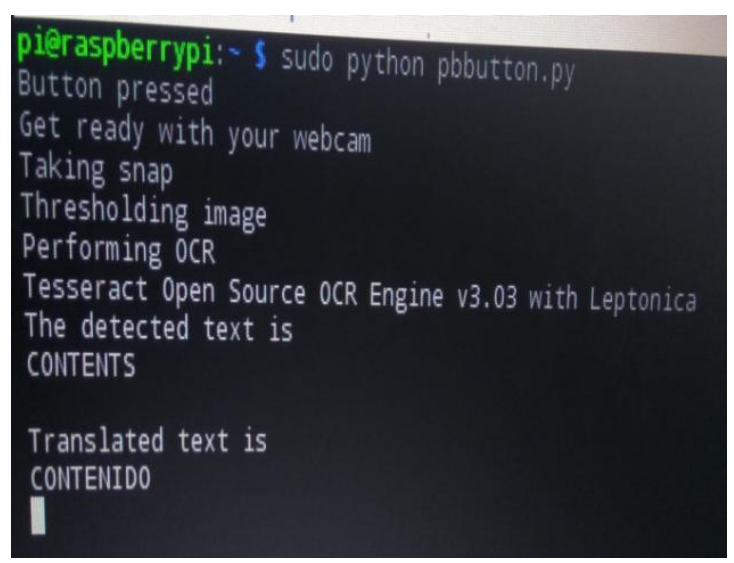

Fig.8 : Program execution-Screenshot

\section{RESULT}

From the present work, the following can be inferred:

- Text is extracted from the image and translated to any desired language.

- It recognizes both capital as well as small letters.

- It recognizes numbers as well.

- Range of reading distance was $38-42 \mathrm{~cm}$.

- Character font size should be minimum $14 \mathrm{pt}$

- Maximum tilt of the text line is 4-5 degree from the vertical.

- The audio of the translated text is outputted.

\section{CONCLUSION}

Text-to-Speech device can change the text image input into sound with a performance that is high enough and a readability tolerance of less than $2 \%$, with the average time processing less than two minutes for A4 paper size. This portable device can be used independently by people. Through this method, international travelers can hear the text images of sign boards, routes in their own languages

\section{REFERENCES}

[1] Archana A, Shinde D. Text pre-processing and text segmentation for OCR. Int.Journal of ComputerScience Engineering and Technology.2012, 2, 810-12.

[2] Simon Monk ,Raspberry Pi Cookbook, published by O'Reilly, 2014

[3] Mithe R, Indalkar S, Divekar N. Optical character recognition.International Journal of Recent Technology andEngineering. $2013,2, .72-75$

[4] Smith R. An overview of the TesseractOCR engine, USA:Google Inc; 2007.

[5] Shah H, Shah A. Optical character recognition of Gujarati numerical. Proc.Int. Conference on Signals, Systems and Automation.2009; 49-53.

[6] Umesh P Image Processing in Python, CSI Communications, December 2012. 